\title{
Identité religieuse et appartenance : une relation d'incertitude
}

À propos de :

Harvey Leonard Patrick, Muslims in Spain, 1500 to 1614, Chicago, The

University of Chicago Press, 2005, 448 p.

\section{Christian Décobert}

\section{(2) OpenEdition \\ 12 Journals}

Édition électronique

URL : http://journals.openedition.org/assr/3446

DOI : 10.4000/assr.3446

ISSN : $1777-5825$

Éditeur

Éditions de l'EHESS

\section{Édition imprimée}

Date de publication : 1 mai 2006

Pagination : 135-145

ISBN : 2-7132-2092-0

ISSN : 0335-5985

Référence électronique

Christian Décobert, «Identité religieuse et appartenance : une relation d'incertitude », Archives de sciences sociales des religions [En ligne], 134 | avril - juin 2006, mis en ligne le 11 juillet 2006, consulté le 19 avril 2019. URL : http://journals.openedition.org/assr/3446 ; DOI : 10.4000/assr.3446 


\title{
Christian Décobert
}

\section{Identité religieuse et appartenance : une relation d'incertitude}

\author{
À propos de : \\ Harvey Leonard Patrick, Muslims in Spain, 1500 to 1614, Chicago, \\ The University of Chicago Press, 2005, 448 p.
}

Après son classique Islamic Spain, 1250 to 1500 (Chicago, 1990), consacré aux stratégies déployées par les musulmans d'Espagne pour préserver l'essentiel d'une culture et d'une pratique islamiques, malgré l'inexorable reconquête chrétienne, L.P. Harvey se penche désormais sur les Moriscos, ceux qui, ayant été convertis de force après la prise de Grenade, continuaient à se vouloir musulmans. C'est l'étude de ces crypto-musulmans, jusqu'à leur expulsion définitive en 1614 , qui est au centre de ce passionnant ouvrage : vie quotidienne, interaction communautaire, pratique religieuse, horizon de culture... Il nous a paru que, grâce à la richesse exceptionnelle des documents qu'il livrait au lecteur, cet ouvrage permettait également de poser la question théorique de l'identité religieuse d'hommes en situation de crypto-appartenance.

Prise de Grenade, 1492, chute du dernier bastion de l'Islam en terre espagnole : désormais tous les musulmans d'Espagne étaient gouvernés par le statut de Mudejars, sujets musulmans ayant accepté la domination chrétienne. Mais ce statut fut de courte durée, quelques années plus tard, des vagues de conversions forcées au christianisme traversèrent la Péninsule ibérique : la Castille, en 15001502 ; la Navarre, en 1515-1516 ; l'Aragon, en 1523-1526. Le mouvement avait débuté au Portugal, en 1497, sur le discours que le royaume était une entité politique gouvernant une unité religieuse : les musulmans portugais n'avaient eu qu'un choix, se convertir ou fuir.

Peu après, les musulmans espagnols furent soumis au même choix. Le prétexte semble avoir été une révolte des musulmans de Grenade et de ses environs, en décembre 1499, qui se soulevaient contre les autorités castillanes accusées de ne pas respecter les termes du traité de reddition les régissant, particulièrement en matière de droit de vivre en tant que musulmans... Une révolte interprétée très différemment, selon l'appartenance religieuse des chroniqueurs : pour le chrétien 
Zurita (XVI siècle), annaliste de la Couronne d'Aragon, il ne s'agissait que d'un mouvement interne aux populations musulmanes, suivi d'un phénomène naturel de contamination. Les musulmans de Grenade, constatant que nombre de leurs coreligionnaires s'étaient convertis, demandèrent à l'archevêque la consécration de leur église - c'est-à-dire la transformation de leur mosquée en église - et les musulmans de la montagne, de guerre lasse, finirent par accepter la conversion. Pour l'historien Al-Maqqārī (vivant au XVII e siècle), les faits sont très différents : il y eut, en premier lieu, violation flagrante du traité entre les Castillans et les musulmans de Grenade et les musulmans d'origine chrétienne furent forcés de se convertir. Les musulmans de souche défendirent avec véhémence les droits de leurs coreligionnaires, mais furent bientôt contraints, eux aussi, de se convertir ou de s'exiler. La plupart d'entre eux partirent vers Fès.

Mais les deux interprétations ne se contredisent pas vraiment : simplement, elles ne se situent pas sur le même plan. Ce que décrit Zurita est une mécanique. Un fait traumatique - ici, une défaite militaire suivie d'une domination " étrangère »- provoque une forte déstructuration sociale, un desserrement des liens communautaires; les populations soumises entrent, dès lors, dans un processus de contamination différentielle, avec des passages à la religion dominante mais, aussi, des îlots de résistance; des conflits ou des tensions intra-communautaires naissent de cette situation contrastée ; jusqu'à ce que s'opère le " ralliement » final des lieux de résistance : Zurita ne s'interroge pas sur la nature de ce ralliement qu'il suppose, nécessairement, sincère. Ce que décrit, en revanche, Al-Maqqārī est un événement, qui permet précisément à cette mécanique de se mettre en marche. Il y a violation des traités, le régime de tolérance et de domination religieuse est contredit : les musulmans de souche chrétienne sont contraints de se convertir au christianisme, alors que les traités garantissaient le culte de tous les musulmans. Mais, pour Al-Maqqārī, cette mécanique est amorcée par un fait totalement nouveau : l'obligation de conversion au christianisme pour les musulmans de Grenade. Plus généralement, l'obligation de se convertir. Ceci lui semble d'autant plus inouï que cette injonction fonctionne, si l'on peut dire, dans les deux sens, c'est-à-dire quelle que soit la religion concernée - christianisme ou islam. Pour comprendre son étonnement, il faut rappeler qu'Al-Maqqårt vit dans l'idée communément partagée que l'homme se situe dans une communauté naturelle d'appartenance - religieuse et sociale. De même que nul n'a été obligé de devenir musulman, nul ne saurait être obligé de devenir chrétien.

Mais précisément, au réflexe d'Al-Maqqārī s'était, par avance, opposé l'argumentaire des hommes de l'Inquisition (et particulièrement l'archevêque Cisneros, de Tolède), pour lesquels la conversion à l'islam de chrétiens d'Espagne s'était faite dans un contexte de domination de l'islam, par conséquent de contrainte, et pour lesquels le retour au christianisme pouvait s'accomplir puisque la domination chrétienne était de nouveau assurée. La mission de l'Inquisition n'étant jamais que l'annulation d'une contrainte et le retour à l'ordre des choses, c'està-dire à la communauté naturelle d'appartenance. 
Dans l'histoire du monde musulman méditerranéen, la reconquête espagnole fut un formidable traumatisme. D'abord, parce que ses ondes de choc se propagèrent dans toutes les régions de l'Islam méditerranéen, par l'exil des élites d'Espagne, à Fès, mais aussi à Tunis, au Caire, à Jérusalem, à Damas, à Alep. Et aussi parce que ce monde musulman méditerranéen se vit désormais entamé : certainement plus que par les Croisades, l'amputation d'une partie de la Maison de l'islam (dār al-islām) sonnait comme la fin d'un monde. Mais la fin de la Reconquista allait provoquer un autre bouleversement...

Avant d'analyser ce bouleversement, rappelons que selon Cisneros, les musulmans anciennement chrétiens relevaient désormais de l'Inquisition à laquelle était assigné un rôle tout à fait nouveau alors que jusqu'alors, qu'elle fût espagnole, portugaise, ou française, elle s'occupait de chrétiens, vieux chrétiens et nouveaux chrétiens (convertis récents au christianisme, du judaïsme notamment) : en tout cas, de chrétiens déclarés. Or Cisneros parlait de musulmans anciennement chrétiens, c'est-à-dire de musulmans déclarés : l'appareil autoritaire de l'Inquisition supposait dès lors l'existence d'une distinction entre une appartenance formelle - qu'elle dénonçait - et une identité religieuse vraie - l'identité chrétienne. Alors qu'auparavant l'Inquisition agissait à l'inverse : admettre une distinction entre une identité religieuse vraie - qu'elle dénonçait (le crypto-judaïsme, par exemple ; ou la démonologie) - et une appartenance formelle - l'appartenance chrétienne : l'appareil inquisitorial semblait entrer ainsi dans un nouvel âge et, en tout cas, se donnait un nouvel objet.

L'Inquisition "ancienne ", née à Rome dès le XIV siècle - et en Castille en 1481 -, posait comme acquise l'appartenance nécessairement chrétienne et c'est l'identité des personnes qu'elle traquait et mettait en procès. Son travail reposait sur l'idée d'une appartenance de nature, et que l'appartenance gouverne l'identité. Puisqu'on est chrétien, on ne peut être crypto-juif ou adorateur de Satan. Cette Inquisition ancienne, qui a bien sûr poursuivi son travail tout au long du $\mathrm{XVI}^{\mathrm{e}}$ siècle, s'est parallèlement donné une autre tâche, renversant complètement les données de ses certitudes. En s'attaquant aux musulmans convertis du christianisme, elle supposait désormais vraie, naturelle, leur identité chrétienne et leur appartenance à l'islam était de facto mise en procès. C'est l'identité qui est de nature, c'est elle qui gouverne l'appartenance : puisqu'on est chrétien - forcément chrétien -, on ne peut appartenir à l'islam. Nous pourrions avancer que là, sur cette articulation, se formulait clairement l'autonomie du sujet croyant. Il y a dans les prémisses réflexifs de ce nouveau travail de l'Inquisition comme une déconnexion ontologique - peut-être définitive - entre identité et appartenance, puisque chacune des deux réalités sert de variable à l'autre, que chacune est l'élément instable qui empêche le recouvrement de l'une par l'autre.

Dans l'Espagne du début du XVI siècle, où un mouvement important de conversion au christianisme a eu lieu, les musulmans manifestement réfractaires ont choisi le chemin de l'exil vers les régions musulmanes. Au sommet de la hiérarchie 
des "anciens musulmans " s'est rapidement reformée une classe de notables locaux, évidemment convertis au christianisme et proches du pouvoir chrétien. Ils occupaient les fonctions d'administrateurs locaux dans les villes d'ancienne domination musulmane, ils défendaient aussi, très directement et très assidûment, les intérêts des "nouveaux convertis ", les conversos. Comme le dit L.P. Harvey, ces notables servaient à la fois leurs nouveaux maîtres et leurs anciens coreligionnaires. Consciemment, les maîtres castillans et aragonais les utilisaient comme médiateurs, garants d'un ordre communément accepté parmi les conversos.

Mais l'on sait que cette classe de notables - et plus largement les populations qui n'avaient pas émigré vers les pays d'islam et qui restaient attachées à ces notables -, étaient, pour beaucoup, des crypto-musulmans. Une abondante documentation a été conservée, montrant que nombreux furent ceux qui restèrent fidèles à leur croyance et à leurs traditions communautaires. Ces documents, qui livrent une information précieuse sur leur vie quotidienne (décrite avec précision et brio par L.P. Harvey), sont rédigés en aljamiado, langue espagnole en caractères arabes. Ce sont des documents cryptés qui circulaient discrètement, utilisés par les conversos crypto-musulmans pour communiquer entre eux, entretenir des réseaux de fidélité et de solidarité, pour circuler et se donner asile réciproque ; mais aussi pour transmettre des éléments d'une culture islamique, jurisprudence, chronique ou observance rituelle. C'est une véritable littérature qui s'est constituée ainsi ; une littérature qui a d'ailleurs fasciné les savants orientalistes dès les débuts de l'orientalisme : le célèbre Silvestre de Sacy, premier professeur d'arabe de l'École Spéciale des Langues Orientales Vivantes (créée par la Convention en 1795) publia deux de ces textes dès 1799 . L.P. Harvey donne à voir, par le commentaire de ces textes, l'étendue d'un fonds juridique, religieux, poétique, que l'on s'efforçait de préserver et de transmettre coûte que coûte.

Ces documents en langue «secrète » n'ont évidemment guère échappé à l'Inquisition. Une Inquisition castillane qui s'est penchée lourdement sur cette population crypto-musulmane et surtout sur ses élites, et qui, à force de procès, d'emprisonnements et de mises à mort, a réussi - avant même la fin du XVI siècle à l'étouffer, à en détruire les réseaux, à casser cette dynamique de transmission d'un savoir et à empêcher toute forme de communication. L'exemple le plus célèbre de ce mouvement pour transmettre le savoir islamique - mais aussi pour l'alimenter par un véritable apport réflexif -, est celui du « Jeune homme d'Arévalo " (El mancebo de Arévalo, né à Arévalo, une ville de Vieille Castille, mais dont on sait qu'il a vécu en Aragon). Sous ce nom, nous sont parvenus plusieurs textes dont le plus important est un Breve compendio de nuestra santa ley y sunna. Harvey pense avoir identifié ce Jeune homme : un certain Agustin de Ribera el mozo, mort dans les geôles de l'Inquisition en 1540 .

Un passage de son Abrégé porte sur le droit de taqiyya (cf.p. 184) : la taqiyya est la dissimulation légale qui gouverne un principe essentiellement shīite, le droit de cacher son identité de shīitite lorsque, propagandiste du shīisme, on 
travaille secrètement à sa diffusion, à sa victoire future. La taqiyya est, en résumé, une « dissimulation stratégique » exactement inverse du quiétisme, comme, par contresens, on a cru parfois la caractériser. Le sunnisme, quant à lui, fait de la taqiyya une situation d'exception, n'acceptant la dissimulation que momentanée; certains juristes prônant un sunnisme rigoriste ont d'ailleurs refusé aux musulmans le droit à la taqiyya. Le sunnisme prône l'obligation première d'être ouvertement musulman.

Le Jeune homme d'Arévalo parle tout autrement de la taqiyya. Prétendant citer un savant ('ālim) du pays d'Espagne, il lui fait dire en substance : nous vivons certes dans une période de terreur, mais Dieu ne manquera pas de nous punir si nous négligeons le service de son royaume. Cependant, le droit de dissimuler nos vraies intentions nous permet de prier sous la forme des chants des chrétiens, dans la mesure où par ces chants les chrétiens recherchent le salut. En conséquence, cette forme de prière peut être considérée comme une dissimulation permise, dans la mesure où une bonne pratique ne peut pas être contredite par une loi rigide. Le Jeune homme commente ainsi le discours du savant : "Voyant des savants aussi respectés, j'étais heureux de vivre et de demeurer en Aragon ". Ces quelques lignes sont formidables de conviction, mais également d'innovation : la dissimulation est ce qui permet de vivre en tant que musulman, c'est-à-dire de servir Dieu, en priant comme les chrétiens, puisque les chrétiens servent Dieu ainsi. Le service de Dieu est indépendant de sa forme, il est dans la simple exigence de se savoir musulman et de prier. Et ceci vaut, pour ainsi dire, comme nouvelle jurisprudence en situation de persécution, contre la tradition d'une loi par trop rigoureuse quant au respect de la forme rituelle. Dans ces mots, le Jeune homme d'Arévalo réinvente la taqiyya, il en fait un état permanent, car sous ces conditions, dit-il, il se déclare heureux de demeurer en Aragon. Pour lui, en définitive, être musulman en secret, c'est s'affirmer musulman, tout en usant de façon durable de la pratique chrétienne, malgré la transgression de la règle qui régit la forme légale du rite musulman.

La question qui se pose est évidemment de savoir si nous n'avons pas, ici, affaire à un cas limite d'acculturation, d'extrême plasticité culturelle, où la substance de ce qui fait l'attache avec le milieu communautaire traditionnel s'évanouit peu à peu pour être remplacée par la substance de ce qui fait le milieu dominant ; et où ce qui reste d'attache est simplement de l'affirmer. Nous serions en présence de ce phénomène "d'espace intervallaire ", au total assez commun aux cryptoappartenances, caractérisé par P.-A. Fabre comme un espace équidistant entre le milieu de tradition et le milieu de domination et d'adhésion nouvelles, un espace qui survit de façon autonome, avec ses modes d'acculturation et ses règles réinventées, mais qui garde la trace de ce qui a fait son passé ${ }^{1}$. Peut-être. Il reste

1. Pierre-Antoine FABRE, « Compte rendu de : Conversioni nel Mediterraneo, sous la dir. de Anna FOA et Lucette SCARAFFIA, Dimensioni e problemi della ricerca storica 2, 1996 », Annales, Histoire, Sciences Sociales, juillet-août 1999, p. 970-973. 
que l'autorité juridique musulmane est venue corroborer le comportement de ce Jeune homme d'Avéralo.

Un texte étonnant apparut dès 1504 (bien avant le Compendio du Jeune homme d'Arévalo) : il s'agit d'une fatwa, c'est-à-dire d'un responsum, consignation d'une réponse juridique à une question posée d'ordre général. Cette fatwa, rédigée à Oran par un mufti, Ahmad Bū Jum'a, inconnu par ailleurs, était destinée aux fidèles proches de Dieu mais vivant hors du domaine de l'islam et qui étaient, clairement, les gens d'Espagne, puisque le texte même parle d'eux avec une évidente sympathie et une certaine acuité - le mufti d'Oran était, à l'évidence, bien informé sur eux. Nous savons d'ailleurs que la fatwa a circulé en Espagne: il existe une copie de sa traduction en aljamiado. La fatwa répondait à une question précise : face à la persécution et à l'obligation de conversion au christianisme, est-ce que des manquements à la sharī $a$, à la loi sacrée, sont tolérés ? En fait, la question en recouvre d'autres, plus délicates à formuler et qui touchent à la crypto-appartenance : peut-on être crypto-musulman ? Si oui, comment ? Quelles sont, dans cette hypothèse, les manières d'être en accord avec la sharī $a$ ? La réponse et l'argumentaire sont pour le moins surprenants, au regard de la jurisprudence de l'époque. Prenons le texte au fil de sa lecture, en le décrivant et en le commentant: il commence par une affirmation d'absolue distinction entre le christianisme et l'islam. Sachez que les idoles ne sont que des morceaux de bois sculpté ou de pierre et qu'elles ne peuvent vous causer aucun tort ni aucun bien, que le royaume est à Dieu seul, qu'il n'a pas de fils, qu'il n'a pas d'associé, qu'il est l'unique que vous devez adorer - le Dieu unique que vous devez adorer dans la persévérance. Après cette courte profession de foi, la fatwa en vient à la description de la pratique croyante en situation de persécution.

Cette pratique est constituée de deux parties : d'abord les rituels, ensuite les interdits. Le ton est donné par la première phrase «vous devez accomplir la prière, même si c'est par un mouvement imperceptible ": nous sommes bien en régime de dissimulation de la pratique. Et la fatwa de passer en revue ce qui fait la prière, pour ce qui est de la forme, de la scansion quotidienne, de l'orientation du priant. Dans l'impossibilité d'observer la forme canonique de la prière, la faire quand même ; si les cinq prières ne peuvent être accomplies en temps voulu, il est préférable de les réciter, si possible, de nuit ; si l'orientation de la Ka'aba ne peut être respectée, il suffit de se donner une orientation symbolique qui la signifierait. Les recommandations sont du même ordre pour ce qui relève des ablutions rituelles: dans l'impossibilité d'utiliser de l'eau, un peu de sable ou une pierre fera l'affaire. Du même ordre encore, mais sur un registre plus subtil concernant le rituel de l'aumône obligée, le texte de la fatwa est très explicite : effectuer le rite de la zakāt (l'aumône obligée du musulman) comme si elle était le geste hypocrite (accompli par le chrétien) de don ostensible à un mendiant, vu par les musulmans comme preuve d'hypocrisie. Bel exemple de pratique de dissimulation où la sincérité d'un geste joue doublement : dénonciation de l'ostentation du chrétien qui fait l'aumône, et principe de non adhésion au geste chrétien 
en simulant son hypocrisie. Mais aussi, jeu d'une réminiscence de la vérité intime du pieux musulman, thématique très prégnante en islam soufi : la piété du musulman sous l'hypocrisie simulée, la sincérité cachée, l'intériorisation extrême du geste rituel et le donner à voir du geste répréhensible, ou à peine tolérable, du « dérèglement " extérieur qui suscite la réprobation générale.

En tout cas, pour ce qui touche aux rites quotidiens du musulman (prière, ablutions, aumône), et pour répondre à la question posée, il est possible de respecter les règles de la sharî ${ }^{-} a$ en situation de persécution : la dissimulation et la simulation sont permises. Mais un leitmotiv revient à chaque ligne dans ce texte : le mot « intention ». Ce qui importe est de prier, de donner en aumône, de faire les ablutions avec intention. L'intention est l'intime, ce qui n'est pas visible.

La fatwa d'Oran est porteuse d'innovation dans la mesure où elle contrevient à toute la littérature jurisprudentielle en islam, à propos de la question de la persécution et de la domination sur des musulmans d'une religion autre, en l'occurrence le christianisme. Jusqu'au Xvl siècle compris, les fatwas, et d'une façon générale la production jurisprudentielle, préconisent l'exil, la hijra. Il n'est, en principe, pas permis de demeurer dans un lieu hostile à l'islam : il faut suivre l'exemple du Prophète Muhammad, qui a quitté La Mecque lorsque lui et ses compagnons ont été persécutés par les membres de la tribu de Quraysh. Cet exil, loin d'être infâme, fut d'ailleurs fondateur, puisque c'est dans l'exil que Muhammad a créé la cité première de l'islam, Al-Madīna et que, pour signifier son importance fondatrice, le temps islamique fut très vite calculé à partir de cette date. Ici, l'obligation d'exil est comme oubliée et fait place à une quotidienneté durable de l'intention intime. La fatwa change de registre : du registre du visible, du musulman qui s'affirme comme tel et qui, là où il est, reconnaît un territoire, le domaine ou la Maison de l'islam (Dār al-islām), au registre de l'invisible, du musulman qui se cache et s'affirme comme ce qu'il n'est pas, et qui, dans sa conviction intime, définit son identité.

Le territoire de l'islam, comme tout territoire religieux, est le lieu d'application de certains interdits. Après avoir passé en revue les questions de rituel, la fatwa d'Oran évoque les interdits. Interdits alimentaires : le crypto-musulman peut boire de l'alcool et manger du porc si, intimement, il les rejette et affirme leur prohibition. Interdits dogmatiques : contraint de dire que Jésus est le fils de Dieu, que le crypto-musulman le dise, avec l'intention de dire que Jésus est le serviteur de Dieu, ou le fils de Marie. Interdits sociaux : tenu de donner sa sœur en mariage à un chrétien, que le crypto-musulman le fasse, avec dans le cœur la conviction d'abhorrer cette action. Ce qui est atteint par ces « dispenses » est ce qui fait lien dans le système communautaire : les prescriptions alimentaires, certes, mais aussi, et surtout, le dogme de l'absolue unicité de Dieu et, enfin, la règle souveraine de l'endogamie religieuse (on ne donne pas les filles hors du groupement musulman, l'aire de circulation des filles circonscrivant exactement l'horizon musulman). C'est la dilution de l'espace communautaire - hérité, reconnu comme 
tel - qui est signifiée par la transgression de ces interdits. Le musulman discret d'Espagne ne recrée son appartenance que sur le ressort de son intention, de ce qu'il a d'intime, de son identité secrète.

La fatwa d'Oran a circulé en Espagne, elle a donc servi et, vraisemblablement, été utile à la génération des premiers crypto-musulmans. Mais on a longtemps pensé qu'elle était un texte unique, circonstanciel, ne représentant pas forcément un consensus de tolérance envers les musulmans d'Espagne, à propos de la non obligation d'exil, de la non observance des rites dans leur forme obligée, du non respect de certains des interdits fondamentaux de l'islam. Mais une découverte récente fait, désormais, taire tout scepticisme. Une autre fatwa, des années 1510 , et hautement "officielle", vient conforter la fatwa d'Oran dans ses réponses. Hautement officielle, car contresignée au Caire par les muftis égyptiens des quatre écoles juridiques de l'islam orthodoxe (malikisme, hanafisme, shāfi'isme, hanbalisme). Cette fatwa ne fait pas du tout référence à la situation espagnole, mais traite de questions liées directement à ce que vivent les musulmans d'Espagne. D'autre part, lieu privilégié d'exil des élites musulmanes venues d'Espagne, il n'est guère pensable qu'on ignorait au Caire l'essentiel de ce qui s'y passait.

Sur plusieurs questions, les avis des quatre muftis divergent quelque peu, mais ils sont strictement convergents sur celles qui relèvent de la situation de musulmans sous domination chrétienne. Trois questions nous intéressent : l'exil, le pèlerinage, et la présence des savants; questions d'ailleurs liées l'une à l'autre. Exil, ou émigration : recommandée, certes, mais à condition qu'elle ne soit pas cause de la ruine de l'émigrant ou des siens : il ne convient pas de dépenser plus du tiers de ses biens en émigrant. Pèlerinage : si un musulman vivant dans un pays sous domination chrétienne parvient à faire le pèlerinage, qu'il revienne là où il réside avec sa famille. Les savants : leur présence est nécessaire pour guider les musulmans là où ils sont, quel que soit le lieu. S'exprime ainsi clairement l'affirmation que l'exil n'est pas l'unique voie possible pour le musulman d'un pays de souveraineté chrétienne, que l'on peut demeurer là où l'on a ses racines et préserver l'espace familial, sans risquer la ruine qu'impliquerait le fait de regagner l'espace de l'islam.

Les trois documents repris de l'ouvrage de L.P. Harvey, du plus personnel au plus officiel (le Compendio du Jeune homme d'Arévalo, la fatwa d'Oran, la fatwa du Caire) que nous avons commentés en les tirant vers les questions d'identité religieuse, sont d'une cohérence lumineuse. Il y eut bien, à l'évidence, un consensus établi sur le droit à la crypto-appartenance. Mais cette cryptoappartenance peut être caractérisée comme une appartenance qui se défait. La forme et le contenu du rite s'oublient dans l'expression de l'intention, les interdits majeurs sont transgressés, le retour dans les terres de l'islam n'est plus une exigence première. Le crypto-musulman ne recrée son appartenance que dans son intime. 
Ce délitement des traits de l'appartenance communautaire et leur perception dans la simple affirmation identitaire n'ont pas surgi soudainement. En d'autres termes, ce possible repli sur l'intime, sur l'identité éminemment personnelle, n'est pas né du dilemme posé par la Reconquête chrétienne de l'Espagne, ni de l'obligation de conversion. L'expression de réponses claires et convergentes au dilemme ainsi posé est, en elle-même, la fin d'un long processus de déconnexion ontologique de l'appartenance et de l'identité.

L'identité religieuse (islām) du musulman (muslim), en tant qu'acte (aslama), a une histoire, l'islam pré-moderne ne fut ni unique ni uniforme. Max Weber distinguait deux âges de l'islam, l'âge guerrier de l'ascèse et l'âge bourgeois du soufisme orgiastique ${ }^{2}$. Nous avancerons simplement ici, pour retourner les choses, que c'est en travaillant, notamment, sur la question de l'appartenance religieuse que l'on peut entrevoir ce qui a distingué ces deux âges. Le premier âge, si on veut le circonscrire chronologiquement, dura jusqu'au XIII ${ }^{\mathrm{e}}$ siècle dans l'Islam dit central ; le deuxième âge, de ce XIII ' siècle axial jusqu'à l'effondrement de l'empire ottoman, au courant XIX ${ }^{e}$ siècle - c'est celui qui nous occupe ici.

Il y a en islam, religion révélée, deux façons de se représenter l'extériorité du fondement et, donc, l'extranéité et l'accessibilité du référent. Les deux référents extérieurs sont la parole de Dieu et Dieu ; leur mode d'accès est le même : l'immédiateté. L'homme d'autorité est celui qui est immédiat à la parole de Dieu ou à Dieu, ou aux deux à la fois ${ }^{3}$. Mais ces deux modes d'autorité ont toujours agi en extrême tension l'un par rapport à l'autre. Ils faisaient tension au sein des appareils sociaux - des savants (immédiats à la parole de Dieu) et des mystiques (immédiats à Dieu) -, comme au sein des dispositifs dogmatiques - sunnisme et shi'isme -, comme ils faisaient tension au cœur même de la pensée de l'homme croyant. Le premier âge de l'islam fut massivement un islam de savants, un islam scripturaire. Le mysticisme islamique, qui fondait l'autorité sur le charisme de l'homme vivant l'union en Dieu, fut d'abord marginalisé, discrédité, pourchassé, mais finit peu à peu par s'imposer, en se structurant, en se donnant, pour dire très vite ce qui demanderait un long développement, une morphologie confrérique. Ce qui se produisit à partir du XIII siècle. Le soufisme, comme forme achevée de mysticisme confrérique, a radicalement transformé l'œkoumène du musulman; il a créé et multiplié les écoles, les mosquées, il a promu le culte des

2. "L'islam fut à ses débuts la religion de guerriers partis à la conquête du monde, d'un ordre de chevaliers, combattants disciplinés de la foi [...]. Mais au cours du Moyen Âge islamique un rôle au moins aussi important a été assumé par le soufisme mystico-contemplatif et par les confréries de la petite bourgeoisie, qui, issues de lui, se sont développées à la manière des tertiaires chrétiens, mais sur un plan beaucoup plus universel ; ce rôle a été assuré sous la direction de techniciens plébéiens de pratiques orgiastiques ", extrait de L'éthique économique des religions mondiales, in Max WEBER, Sociologie des religions, éd. Jean-Pierre GROSSEIN, Paris, Gallimard, 1996, p. 334.

3. Christian DÉCOBERT, "L'autorité religieuse aux premiers siècles de l'islam », Archives de Sciences Sociales des Religions 125, 2004, p. 23-44. 
saints et parsemé le monde musulman de tombes vénérées : un nouveau paysage se créait, produit par les milieux confrériques. Le soufisme se diffusait partout, il quittait les milieux lettrés, s'urbanisait et se ruralisait, il constituait désormais une culture commune dans les territoires de l'Islam central, puis dans ceux de l'Islam dit périphérique. Max Weber a dit vrai : le deuxième âge de l'islam fut massivement celui du soufisme. Ce qui ne revient pas à dire que le mystique dépossédait le scripturaire, loin s'en fallait, mais que le rapport de tension qui les liait l'un à l'autre était affecté d'une variable nouvelle, selon laquelle la pratique mystique était désormais une dominante sociale.

La diffusion exponentielle du soufisme banalisa et installa, dans les reins et les cours, une donnée nouvelle : le soufisme comme vecteur de la généralisation de l'expérience de l'intime, qu'engageait la pratique mystique. De fait, une telle pratique permet de générer le principe selon lequel la vérité du musulman se trouve dans l'intime. Dans son parcours initiatique - qui prend des formes très diverses et très populaires (danses, chants, oraisons répétitives, etc.) - l'homme musulman découvre que c'est dans le rapprochement intime du divin (dans le désir de fusion avec Dieu) qu'il trouve sa vérité d'homme musulman. Mais c'est, par là même, son identité de musulman qui se recompose. Celle-ci n'est plus, seulement, une identification communautaire, elle est désormais la composition de deux éléments qui sont à la fois l'intime et le communautaire. Là réside une identité personnelle nécessairement composite. L'énorme corpus de littérature soufie le montre de façon suffisamment claire : la procédure initiatique est à la fois un cheminement vers l'intime, vers le cœur de soi et donc vers Dieu, et un évidement de soi. Mais cet évidement de soi ne laisse pas un vide qui, pour ainsi dire, se remplirait uniquement de Dieu : un tel vide se remplit, aussi, de ce qui est intrinsèquement communautaire, de toutes les valeurs traditionnelles du communautaire, lesquelles sont, en somme, reformulées, intériorisées.

Mais si l'identité personnelle est ce recouvrement absolument nécessaire de l'intime et du communautaire, s'il y a nécessité de recouvrement, ce fait même induit, en toute règle ontologique, qu'existerait possiblement le « non recouvrement » de l'intime et du communautaire. Auquel cas le musulman ne serait pas un vrai musulman. Les conséquences en sont évidemment nombreuses. Dès lors, il est par exemple permis de discuter de l'authenticité de l'appartenance musulmane. Et ce n'est pas un hasard si les milieux soufis se chargèrent de l'évaluation. $\mathrm{Si}$, par exemple, les gouvernants se conduisent mal, n'est-ce pas parce que, intimement, ils ne sont pas de vrais musulmans ? Là est la raison de la dénonciation du gouvernant impie. Mais, par ailleurs, il faut comprendre que si cette nouvelle définition de l'identité musulmane en islam pose l'intime au centre de l'homme croyant, c'est-à-dire exhibe la conviction, cela ne signifie nullement qu'il y aurait dissociation entre identité personnelle et appartenance à la communauté naturelle d'appartenance. Bien au contraire, il s'agit d'un rattachement du communautaire à l'intime. Et, de fait, l'histoire sociale nous montre que les liens du 
communautaire furent considérablement resserrés sous le règne des réseaux de confréries mystiques. Les milieux soufis furent les agents de ce resserrement, en constituant les réseaux confrériques sur une exacte adéquation avec les principes communautaires - notamment les règles du lignage et de la cohésion collatérale.

Ce que les mouvements de Réforme, mais aussi, et largement, les pratiques de l'Inquisition, ont transformé dans le monde chrétien, le soufisme confrérique l'a transformé dans le monde islamique: une représentation de l'identité de l'homme croyant, où l'intime devient central. Pourtant, ce moment d'invention de l'intime, ce tournant préjudiciel dans l'histoire de l'islam se trouva comme une fin ultime - nous pourrions dire, comme une fin logique - dans l'horizon possible du délitement des instances du communautaire, et des règles formelles de l'appartenance. C'est sur cette suspension, en quelque sorte, que l'islam méditerranéen allait vivre les trois siècles de la domination ottomane. Jusqu'au XIX siècle, où tout fut remis en question, et où l'on vit « le communautaire " ressurgir avec quelque violence. Mais ceci ouvre à un autre âge de l'islam.

Christian DÉCOBERT CNRS - Centre d'études interdisciplinaire des faits religieux 